Note: This is a draft of a paper submitted for publication. Contents of this paper should not be quoted or referred to without permission of the author(s).

To be submitted to the Materials Research Society Fall Meeting 1999, Boston, MA November 29 - December 3, 1999.

\title{
Z-Contrast STEM Imaging and Ab-Initio Calculations of Grain Boundaries in $\mathrm{SrTiO}_{3}$
}

\author{
M. Kim, ${ }^{1,2}$ N. D. Browning, ${ }^{1}$ S. J. Pennycook, ${ }^{2}$ K. Sohlberg, ${ }^{2}$ S. T. Pantelides ${ }^{2,3}$ \\ ${ }^{1}$ Department of Physics, University of Illinois at Chicago, Chicago, $\mathbb{L}$ \\ ${ }^{2}$ Solid State Division, Oak Ridge National Laboraotry, Oak Ridge, TN \\ ${ }^{3}$ Department of Physics and Astronomy, Vanderbilt University, Nashville, TN
}

The submitted manuscript has been authored by
a contractor of the U.S. Government under
contract No. DE-ACO5-960R22464.
Accordingly, the U.S. Government retains a
nonexclusive, royalty-free license to publish or
reproduce the published form of this
conribution, or allow others to do so, for U.S.
Government purposes.".

\author{
prepared by \\ SOLID STATE DIVISION \\ OAK RIDGE NATIONAL LABORATORY \\ Managed by \\ LOCKHEED MARTIN ENERGY RESEARCH CORP. \\ under \\ Contract No. DE-AC05-96OR22464 \\ with the \\ U.S. DEPARTMENT OF ENERGY \\ Oak Ridge, Tennessee
}




\title{
Z-Contrast STEM Imaging and Ab-Initio Calculations of Grain Boundaries in $\mathrm{SrTiO}_{3}$
}

\author{
MIYOUNG KIM ${ }^{1.2}$, NIGEL D. BROWNING', STEPHEN J. PENNYCOOK ${ }^{2,3}$, KARL $^{1}$ \\ SOHLBERG $^{2}$, SOKRATES T. PANTELIDES ${ }^{2.3}$ \\ 'Department of Physics, University of Illinois at Chicago, Chicago, IL; \\ ${ }^{2}$ Solid State Division, Oak Ridge National Laboratory, Oak Ridge, TN; \\ ${ }^{3}$ Department of Physics and Astronomy, Vanderbilt University, Nashville, TN.
}

\begin{abstract}
The understanding of electrical properties of grain boundaries in perovskites is essential for their application to capacitors, varistors and positive-temperature coefficient resistors. The origin of the electrical activity is generally attributed to the existence of charged defects in grain boundaries, usually assumed to be impurities, which set up a double Schottky barrier as they are screened by dopants in the adjacent bulk crystal. Microscopic understanding of the origin of the grain boundary charge, however, has not been achieved. It is not known yet if the charged grain boundary states are an intrinsic property of a stoichiometric grain boundary, arise from nonstoichiometry, or are caused by impurities. Here, the relation between atomic structure and electronic properties is studied by combining experiment with ab-initio calculations. The starting structures for theoretical calculations were obtained from Z-contrast images combined with electron energy loss spectroscopy to resolve the dislocation core structures comprising the boundary. Dislocation core reconstructions are typical of all grain boundaries so far observed in this material. They avoid like-ion repulsion, and provide alternative sites for cation occupation in the grain boundaries. Optimized atomic positions are found by total energy calculations. Calculated differences in vacancy formation energies between the grain boundaries and the bulk suggest that vacancy segregation can account for the postulated grain boundary charge.
\end{abstract}

\section{INTRODUCTION}

Transition-metal perovskites such as $\mathrm{SrTiO}_{3}$ and $\mathrm{BaTiO}_{3}$ have been extensively studied both theoretically and experimentally because of their interesting structural, optical, and electronic properties, which allow many applications for various electronic devices as dielectrics and ferroelectrics [1-3]. Grain boundaries are known to be responsible for their transport properties such as nonlinear I-V characteristics, so the efficiency of devices critically depends on the microstructure of interfacial defects, especially of grain boundaries. The potential barrier at the grain boundaries has been explained phenomenologically as a back to back double Schottky barrier[4-6]. The origin of the barrier has been attributed to the presence of immobile charge on the grain boundary plane. This charge is compensated by ionized impurities in the bulk, giving rise to a space charge depletion layer. The total space charge is equal to the excess charge at the interface, to maintain overall charge neutrality, and is related to the electrostatic potential by the Poisson equation. In equilibrium, a potential barrier is set up which opposes the passage of free carriers through the grain boundary.

The atomic-scale origin of this charged boundary state, however, has not so far been explained. It may be produced by a segregation of defects such as dopants, interstitials, or vacancies. In n-type materials, for example, segregation of cation vacancies or electrons trapped at deep acceptor states will lead to a potential barrier. Correspondingly, in p-type materials, a barrier will arise from segregation of anion vacancies or excess cations. It is therefore necessary to study the microscopic atomic and electronic structure of the grain boundaries to understand their macroscopic electrical properties. Here, we present atomic resolution Z-contrast images of grain boundaries in $\mathrm{SrTiO}_{3}$ and ab-initio theoretical calculations of both stoichiometric and nonstoichiometric boundary structures to investigate the microscopic origin of the interface charge. 

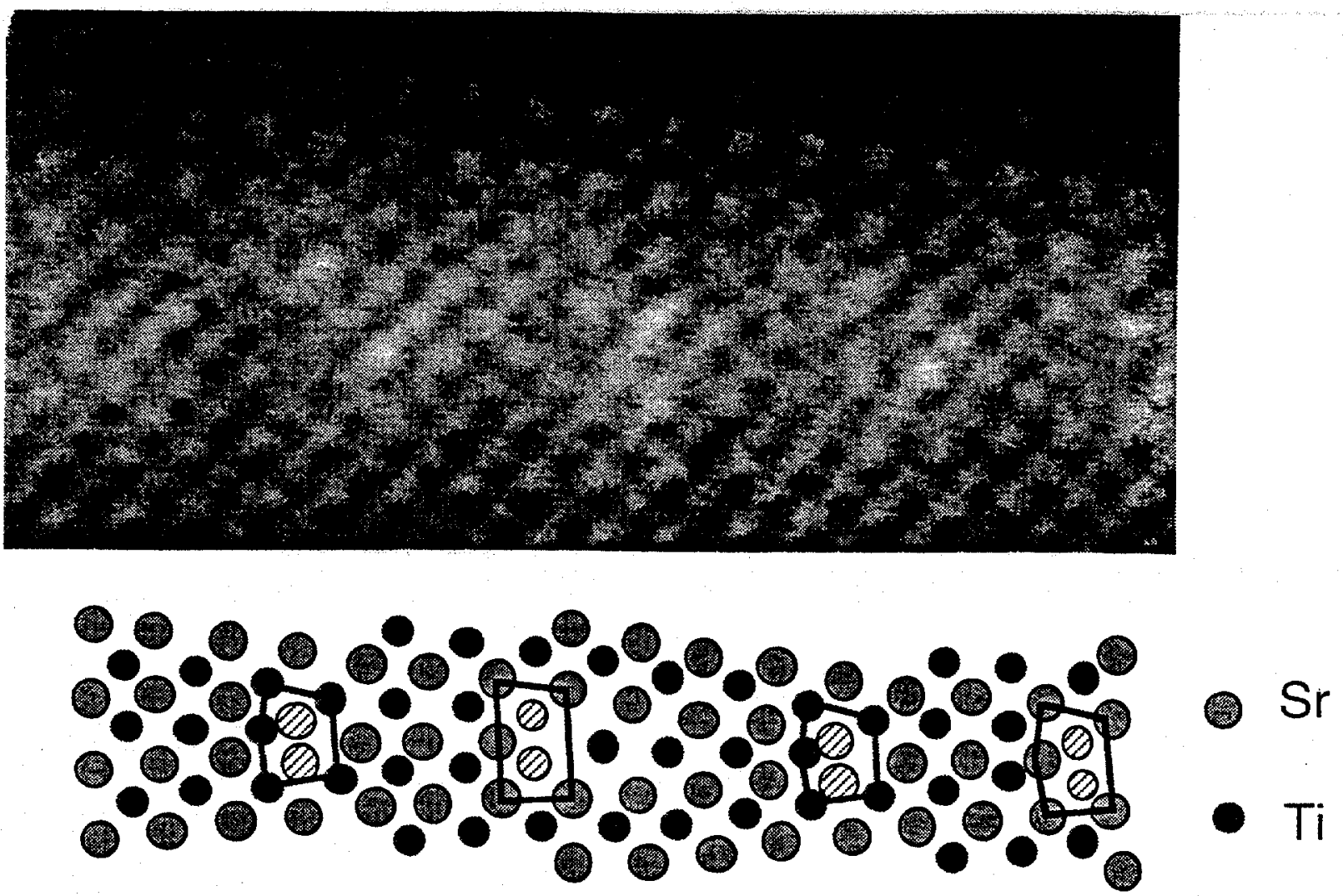

Figure 1. Z-contrast STEM image of a $15^{\circ}$ [001] grain boundary, which shows both titanium and strontium dislocation cores.

\section{EXPERIMENT}

The geometric structure of $\mathrm{SrTiO}_{3}$ grain boundaries has been obtained from Z-contrast STEM images[7-9]. The dark field image obtained from a high angle annular detector is incoherent, so the image is described as convolution of the atomic scattering cross section with the STEM probe profile, which allows direct location of atomic columns from the image without extra simulations. The intensity of each atomic column is directly related to the atomic number, so it is possible to deduce both structure and composition. The ability to distinguish Sr from $\mathrm{Ti}$ sites is especially useful in the case of interface or grain boundary structures. Figure 1 shows a Z-contrast image from a $15^{\circ}<001>$ tilt grain boundary in $\mathrm{SrTiO}_{3}$ obtained with the VG Microscopes HB603U STEM which has a probe size of $1.3 \AA$. It resolves the dislocation core structures comprising the boundary, showing the reconstructions which are typical of all grain boundaries so far observed in this material. As shown in the schematic, the two atomic columns in the centers of each dislocation core are spaced only $2.5 \AA$ apart. Ionic repulsion clearly excludes the possibility of both sites being simultaneously occupied in any single atomic layer normal to the tilt axis. Therefore, these two sites are alternate sites for cation occupation, and the core is reconstructed.

While the high-angle scattered electrons form a dark field image, the small-angle scattered electrons are passed through a spectrometer to provide an electron energy loss spectrum (EELS). EELS provides complementary information for distinguishing atomic columns in addition to $Z$ contrast image, especially light atoms which are not detected in Z-contrast images. EELS is also sensitive to changes in electronic structure or valence induced by changes in local coordination [10]. 


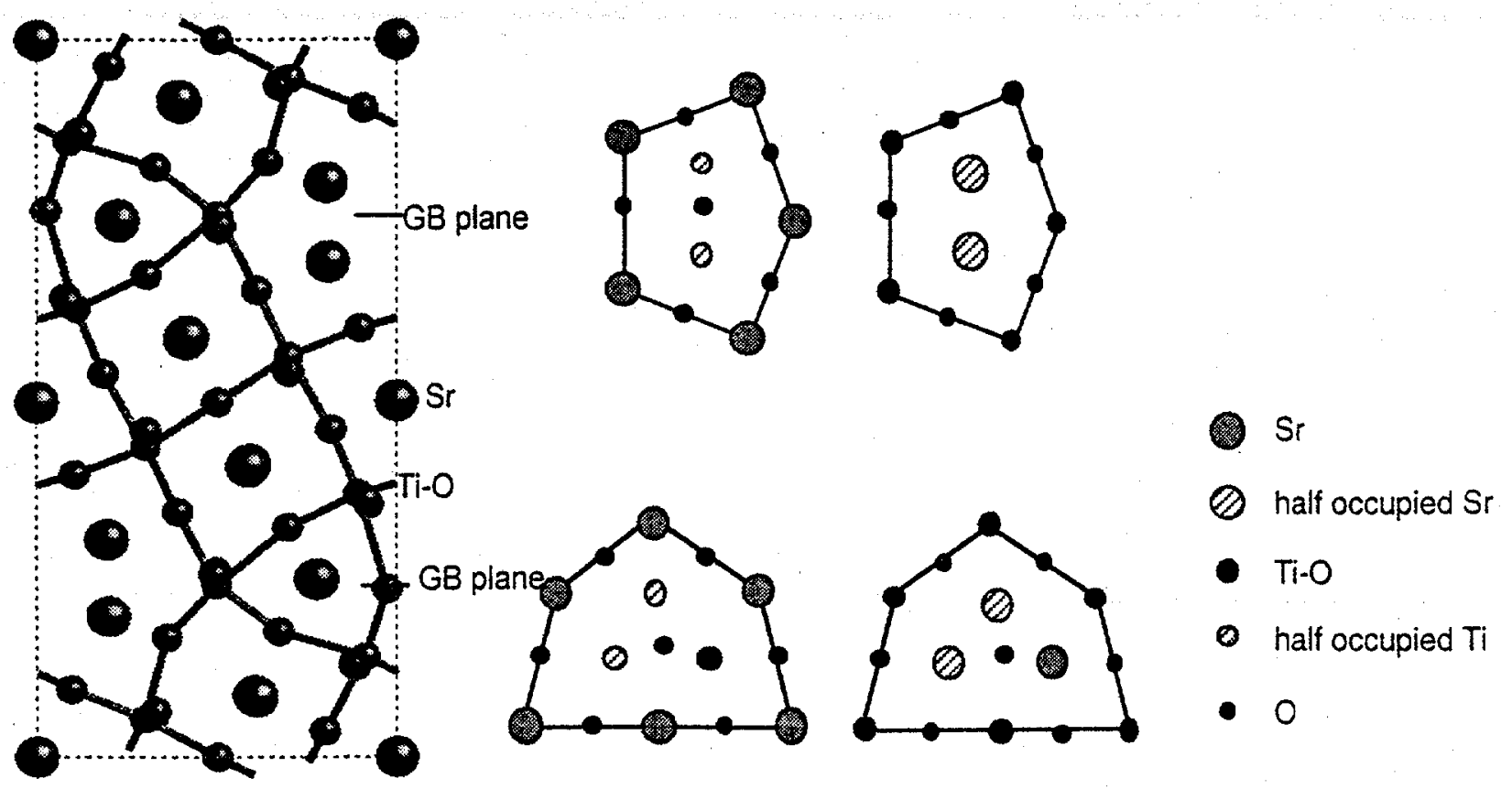

Figure 2. The relaxed supercell with two $53^{\circ}$ symmetric $\{210\}$ grain boundaries and dislocation cores observed in $\mathrm{SrTiO}_{3}$ grain boundaries.

\section{CALCULATIONS}

We performed self-consistent ab-initio density functional calculations using density functional theory, the local-density approximation for exchange-correlation, pseudopotential and plane waves. Supercells are set up containing two grain boundaries in order to allow periodic boundary condition. Ultrasoft pseudopotentials were used with a plane wave energy cutoff of 380 $\mathrm{eV}$, and the Monkhorst-Pack scheme was used for Brillouin zone sampling[11]. In titanium, 3s and $3 p$ states were treated as semicore states, and the strontium pseudopotential included $4 s$ and $4 p$ electrons. The all band mixing scheme is used for electronic minimization, and the ionic relaxation used the Broyden-Fletcher-Goldfarb-Shanno scheme[12]. The exchange-correlation potential is represented by the Ceperley-Alder form. Figure 2 shows a relaxed supercell with two $53^{\circ}$ symmetric $\{210\}$ grain boundaries and its constituent unit. The relaxation was continued until the residual force reached $0.05 \mathrm{eV} / \AA$, and the residual energy reached $0.5 \mathrm{E}-5 \mathrm{eV} / \AA$. In bulk $\mathrm{SrTiO}_{3}$, the experimentally obtained lattice constant is $3.905 \AA$ while $3.863 \AA$ was obtained by the ab-initio calculation. The lattice constants used for reconstructed $\mathrm{SrTiO}_{3}$ supercell, $\mathrm{a}=8.637 \AA$, $b=18.115 \AA, c=7.725 \AA$, were based on the theoretically obtained lattice constant of the bulk crystal. The grain boundaries are allowed to relax and expand in the b-direction, and the atoms in the bulk layer between the two grain boundaries are fixed to prevent the direct interaction between two grain boundaries.

Although strontium and titanium are seen in the image of Fig. 1, the oxygen columns are not seen because of their much lower intensity. The number of oxygen atoms is chosen to preserve stoichiometry. Initial positions were chosen as in the schematic of Fig. 2. For nonstoichiometric grain boundary calculations, this supercell size was fixed at constant volume, and all the criteria remained the same.

After we obtained the optimized geometric structure, we used the full potential linearized augmented plane wave (FLAPW) method to obtain the electronic structure of each equivalent atomic column of this geometry[13]. The FLAPW method includes all electrons, and treats the 


\section{Stacking fault}

(001)

- Oxygen

- $\mathrm{Ti}$

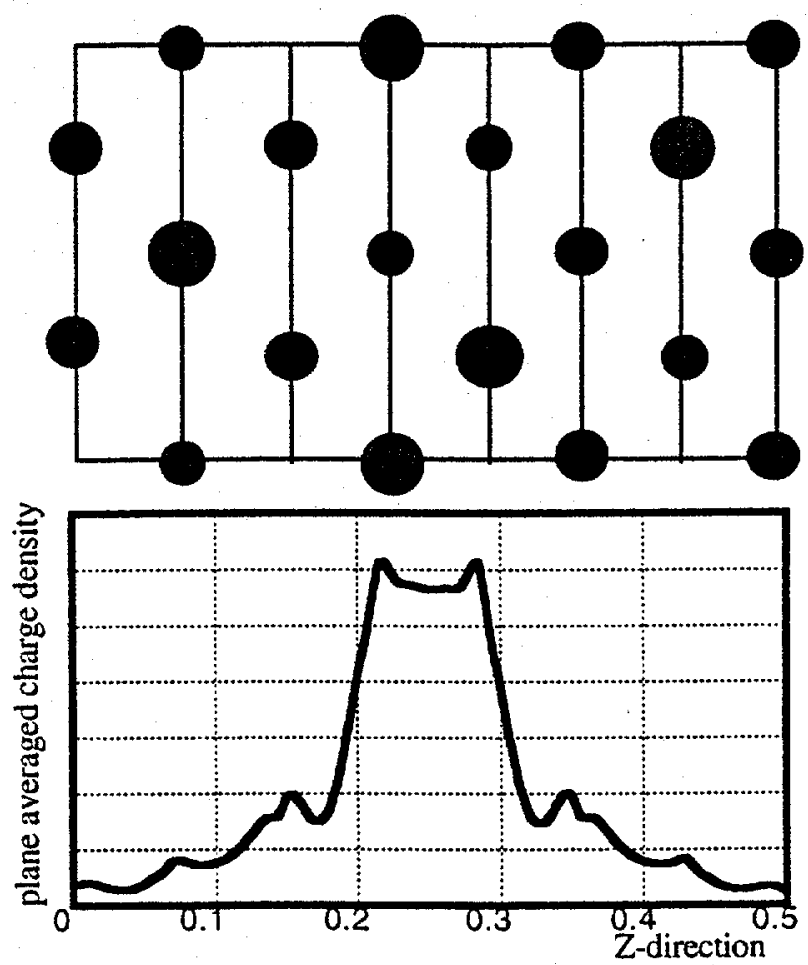

Figure 3. One half of a unit cell containing two stacking faults, and the corresponding planeaveraged charge density in the conduction band.

core and valence electrons separately. The basis sets for the inside and the outside of the muffin tin are spherical harmonics and plane waves respectively. The exchange-correlation potential was also the local density approximation. The number of $k$-points used to expand the crystal reciprocal vector was 48 , and the muffin-tin radius of each atom was $2.0,1.6,1.6$ atomic units (a.u.) respectively. The self-consistency criterion for the charge difference was 0.001 between the old and new charge distributions.

\section{RESULTS}

First, we compare the total energy of the reconstructed and unreconstructed stoichiometric grain boundaries. In the case of the grain boundary constructed from $\mathrm{Sr}$ cores, the reconstruction into alternating strontium columns shown in Fig. 2 is energetically favorable by $0.5 \mathrm{eV} / \mathrm{cell}$ compared to an unreconstructed boundary. The formation energy for the reconstructed strontium grain boundary is $14.6 \mathrm{eV} / \mathrm{cell}$. The experimentally observed energy band gap is $3.2 \mathrm{eV}$ while the calculated energy band gap is $2.0 \mathrm{eV}$ in the bulk and $1.8 \mathrm{eV}$ in the grain boundary supercell. The decrease of the band gap may be explained by the increase of orbital overlap in the grain boundary region due to lattice misfit [14]. There is no deep level in the band gap, which suggests the stoichiometric grain boundary may not be responsible for the potential barrier.

In order to illustrate the essential physics of the origin of electrical activity of grain boundaries we performed initial calculations on a stacking fault which exhibits an excess of cations (equivalently, oxygen deficiency). The concomitant valence imbalance leads to excess electrons that must go into the conduction bands or "donor" bands pulled out of the conduction bands. First-principles density functional calculations for this system illustrate the effect. In figure 3 , we show the density of the excess electrons in the conduction band region. The amplitude is primarily 
on the cation sites, which is consistent with the fact that the lowest conduction bands are made up of cation orbitals, and decays with distance from the grain boundary. These electrons would create a double Schottky barrier. In an acceptor doped material for example, they would migrate to nearby ionized acceptors losing a significant fraction of the band gap energy in the process. The acceptors would then be negatively charged, thereby establishing the space charge necessary to screen out the undercoordinated, positively charged cations at he grain boundary. Note that the system contains no vacancies or free volume. Although this stacking fault structure may be unstable, we use it here only to illustrate the effect of excess cations, or equivalently, oxygen vacancies on the energy bands. A calculation for an oxygen vacancy in an otherwise perfect crystal produced a very similar effect with electrons in states near the conduction-band edge.

Calculations of the oxygen vacancy segregation energy were then performed for the grain

boundary structure of Fig. 2 . Here, the segregation energy is defined as the difference in vacancy formation energy between the bulk and the grain boundary. For the bulk calculation, one oxygen in 8 unit cells was removed. In the case of the grain boundary, two different sites were selected, as shown in figure 1. Removing one of the two oxygen atoms in each column resulted in an oxygen vacancy segregation energy of $3.5 \mathrm{eV}$ for site (a) and $3.6 \mathrm{eV}$ for site (b). When we removed both oxygen atoms in a column (ie. the entire column), the segregation energy decreased to $1.0 \mathrm{eV}$. These calculations therefore suggest that oxygen vacancies are attracted to the grain boundary in concentrations below $50 \%$, where they would establish the non-stoichiometry required to explain the origin of the double Schottky barrier.

\section{ACKNOWLEDGMENTS}

This research was sponsored by the Division of Materials Sciences, U.S. Department of Energy, under contract DE-AC05-96OR22464 with Lockheed Martin Energy Research Corp., by NSF under grant no. DMR-9803021, NSF under grant DMR-9803768, and by an appointment to the ORNL postdoctoral research program administered jointly by ORNL and ORISE.

\section{REFERENCES}

1. T. Wolfram. Phys.Rev.Lett. 29, 1383 (1977)

2. V.E. Henrich, Rep. Prog. Phys, 48, 1481 (1985)

3. M. Vollman and R. Waser, J. Am. Ceram. Soc. 77 235-243 (1994)

4. K.L. Kliewer and J.S. Koehler, Phys. Rev, 140, pp. A1226-A1240 (1965)

5. W.E. Taylor, N.H. Odell, and H.Y. Fan, Phys. Rev, 88, 867-875 (1952)

6. J.A.S. Ikeda and Y. -M Chiang, J. Am. Ceram. Soc. 76, 2437-2446 (1993)

7. M.M. McGibbon, N.D. Browning, M.F. Chisholm, A.J. McGibbon, S.J. Pennycook, V. Ravikumar, and V.P. Dravid, Science, 266102 (1994)

8. N.D. Browning, S.J. Pennycook, M.F. Chisholm, M.M.McGibbon, and A.J. McGibbon, Interface Science, 2, 397-423 (1995)

9. M. M. McGibbon, N.D. Browning, A.J. McGibbon, and S.J. Pennycook, Philosophy Mag. A, 73, 625 (1996)

10. G. Duscher, N. D. Browning and S. J. Pennycook, Phys. Stat. Sol. A 166, 327 (1998)

11. H.J. Monkhorst and J.D. Pack, Phys. Rev. B 13, 5188 (1976)

12. M.C. Payne, M.P. Teter, D.C. Allan, T.A. Arias, and J.D. Joannopoulos, Rev. Mod. Phy., 63,1045 (1992) 
13. P. Blaha, K. Schwarz, and J. Luitz, WIEN97, Vienna University of Technology 1997

14. I. Dawson, P.D. Bristowe, M.-H. Lee, M.C. Payne, M.D. Segall, and J.A. White, Phys. Rev. B, 54, 13727-13733 (1996) 